\title{
Special session on HD 5980
}

\author{
Anthony F.J. Moffat ${ }^{1}$ \\ Département de physique, Université de Montréal, \\ C.P. 6128, Succ. Centre-Ville, Montréal, Qc H3C 3J7, Canada
}

\begin{abstract}
On Monday before the regular meeting began, a group of 31 participants met for 5 hours to discuss HD 5980, a massive binary in which one of its components erupted in 1994 as a luminous blue variable (LBV). This made HD 5980 the most luminous star in all the SMC for about six months. The highlights of this session are presented here.
\end{abstract}

\section{Highlights}

\subsection{Among the issues that most participants agreed on}

1. The observed spectrum changed slowly at first, then more rapidly, from WNE+O in the 1970's through WN6, WN7, WN8 to WN11 around maximum light, when HD 5980 had brightened by 3 full magnitudes.

2. The eruption occurred in star A that is defined to be the component that passes in front at primary light-minimum in an eclipsing binary system with period of 19.265 days and moderate ellipticity $e=0.27$. Star B is the component that passes in front at secondary minimum at phase 0.36 .

3. This is the first known case of an LBV eruption of a massive star in a very close binary, with important potential consequences for mass exchange in rapidly evolving binaries.

4. Although HD 5980 lies close to the SMC's youngest massive cluster NGC 346, it does not appear to be directly related in any obvious way to the cluster. Along with the nearby O7Iaf+supergiant Sk 80, HD 5980 (= Sk 78) probably belongs to a somewhat older, spatially extended population of field OB stars.

\subsection{Among the issues still unclear}

5. Although the orbital shape is known fairly well, its size (based on the poorly known radial velocity amplitudes) is not. Nevertheless, both stars must be massive, with current masses in the range of $\sim 30-80 \mathrm{M}_{\odot}$ for star $\mathrm{A}$ and $\sim 10-30 \mathrm{M}_{\odot}$ for star B.

6. The true spectral types of the stars before the eruption remain elusive. Star A may be a hot, luminous Of star, while star B may be a relatively hydrogen-poor

\footnotetext{
${ }^{1}$ Killam Fellow of the Canada Council for the Arts
} 
WN star. Alternatively, both orbiting stars might be WNE, based on RV data from photographic spectra for the very weak and variable N IV 4058 and $\mathrm{N} \mathrm{V}$ 4603 emission lines that appeared to move in antiphase. In the latter case, the photospheric absorption lines seen before the eruption must have come from a third star, whose presence is a subject of debate. The former scenario, in which an evolved $\mathrm{O}$ star goes LBV, is more orthodox than the second scenario, in which a WNE star goes LBV. The jury is still out, though, since no-one knows what really can happen at the $0.1 \mathrm{Z}_{\odot}$ of the $\mathrm{SMC}$.

7. Why did star A erupt only to WN11, like the LBV AG Carina at minimum light? Perhaps HD 5980 went further without having been noticed, since there were gaps in the observations around maximum light, or perhaps the eruption was "throttled" by the presence of a close companion.

8. During and after the eruption, double-line emission profiles were seen, implying that mass may be leaving the system. It is not clear how much mass left the system as a result of the eruption, although it is unlikely to be more than the deduced mass loss from the spectrum of the erupting star, $0.001 \mathrm{M}_{\odot}$. The change in orbital period expected (maxiumum 1 part in $10^{5}$ ) was not detectable.

9. Does line emission from colliding winds dominate the pre-eruption spectrum? This appears to be a viable explanation for the spectacular phase-dependent line profile variations in the pre-eruption spectrum, although other explanations cannot be entirely excluded. Energy requirements to produce such high line-flux from wind collision may or may not be a problem, depending on the detailed history of the collision shock process, which must have been different from what occurs in the WR wind.

\subsection{What next?}

In an attempt to resolve some of these issues, coordinated campaigns on HD 5980 should be pursued. Two such campaigns were suggested:

10. Long-slit spectroscopy with $H S T$ to search for ejecta in the immediate surroundings of HD 5980, and if the system returns to $\mathrm{WN}+\mathrm{O}$, search for the photospheric lines of star A (and B?) and a possible 3rd component in the system.

11. Observe NGC 346 along with HD 5980 using the high-resolution X-ray imager on $A X A F$, in search of interacting winds.

Acknowledgments. I thank Gloria Koenigsberger for helping to organise this session.

\section{Participants}

Jane Arthur, Jacques Breysacher, Jorge Cantó, Mike Corcoran, Luis Corral, Paul Crowther, Orsola De Marco, Sean Dougherty, Aaron Flores, Guillermo García-Segura, Leonid Georgiev, Eric Gosset, Lex Kaper, Gloria Koenigsberger, Chris Lloyd, Siegfried Lührs, Sergey Marchenko, Divakara Mayya, Tony Moffat, Virpi Niemela, Julian Pittard, Francesco Polcaro, Corinne Rossi, Pierre Royer, Werner Schmutz, Mike Shara, Ian Stevens, Dany Vanbeveren, Karel van der Hucht, Nolan Walborn, Debra Wallace. 